\title{
Quantifying pulmonary perfusion in primary pulmonary hypertension using electron-beam computed tomography
}

\author{
A.T. Jones*, D.M. Hansell ${ }^{\#}$, T.W. Evans*
}

Quantifying pulmonary perfusion in primary pulmonary hypertension using electronbeam computed tomography. A.T. Jones, D. M. Hansell, T.W. Evans. C) ERS Journals Ltd 2004.

ABSTRACT: Traditionally, a gravitational distribution of pulmonary perfusion has been described in normal subjects. How this may vary in patients with primary pulmonary hypertension (PPH), which is characterised by vascular obstruction due to intimal thickening, smooth muscle cell proliferation and episodes of thrombosis in small and medium sized pulmonary arteries, is unclear. In this study the potential of electronbeam computed tomography in quantifying the distribution of pulmonary perfusion in patients with PPH was investigated.

Contrast-enhanced sections were obtained during inspiration in the supine position at baseline and during administration of the vasodilator adenosine in five healthy subjects and five patients with PPH. Under each experimental condition, regions of interest were placed along the nondependent-to-dependent axis and values for relative perfusion derived.

In healthy individuals, a marked nondependent-to-dependent gradient in perfusion was observed. By contrast, in PPH, perfusion values were significantly lower and were uniform across the lung section, although the administration of adenosine resulted in increased perfusion in all regions of interest.

Electron-beam computed tomography provides physiological and structural information about the pulmonary circulation in subjects with pulmonary vascular disease. Eur Respir J 2004; 23: 202-207.
*Unit of Critical Care, and ${ }^{\#}$ Dept of Imaging, National Heart and Lung Institute, Imperial College of Science, Technology and Medicine, Royal Brompton Hospital, London, UK.

Correspondence: T.W. Evans

Royal Brompton Hospital

Sydney St

London

SW3 6NP

UK

Fax: 441713518524

E-mail: t.evans@rbh.nthames.nhs.uk

Keywords: Computed tomography pulmonary hypertension pulmonary perfusion

Received: March 262003

Accepted after revision: August 52003
Traditionally, a gravitational distribution of pulmonary perfusion has been described in normal subjects, determined by the interrelationship between hydrostatic, alveolar and interstitial pressures [1, 2]. More recently, animal studies, employing techniques with high spatial resolution, have suggested that the influence of gravity is less important than the structure of the pulmonary vascular tree in determining regional blood flow in the lungs [3]. Similar findings in humans have been confirmed by a study employing electronbeam computed tomography (EBCT) to quantify regional pulmonary perfusion [4]. EBCT allows the fast (millisecond) acquisition of the data necessary for cardiac imaging. Pulmonary regional blood flow can be calculated by applying an appropriate model to changes in lung density detected, following the passage of contrast. EBCT has been used previously in both animal and clinical studies of perfusion [5-7], the results obtained showed a good correlation with those found from microsphere-based investigations.

Primary pulmonary hypertension (PPH) is characterised by the restriction of the pulmonary vascular bed, secondary to a combination of intimal thickening, vascular smooth muscle cell proliferation and episodes of thrombosis, in small- and medium-sized arteries. Studies of pulmonary perfusion patterns in PPH, excluding those aimed at differentiating between PPH and chronic thromboembolic disease, are scarce. However, investigations using computed tomography (CT) have shown that patients with systemic sclerosis and secondary pulmonary hypertension have a reduced density gradient between the dependent and nondependent parts of the lung, compared with similar patients without elevated pulmonary vascular resistance [8]. Secondly, using technetium-99m-labelled, macroaggregated albumin, the gravitational gradient in perfusion has been shown to be more uniform in patients with PPH compared with normal subjects, in both erect and supine positions [9]. Similar findings have been reported using positron emission tomography (PET) [10].

In this study EBCT was used to study regional pulmonary perfusion in patients with $\mathrm{PPH}$ and to assess changes in response to the intravenous infusion of adenosine, a shortacting vasodilator routinely used in trials of pulmonary vascular reversibility [11]. For comparison, perfusion was also studied in five male, nonsmoking, healthy subjects.

\section{Methods}

The experimental protocol, to which all subjects provided written and informed consent, was approved by the Ethics Committee of the Royal Brompton Hospital and was designed to minimise radiation exposure. The effective dose for each subject was $2.2 \mathrm{mSv}$ for subjects with PPH and $1.1 \mathrm{mSv}$ for normal subjects $(\sim 25$ and $12.5 \%$ of the effective radiation dose given as conventional, contiguous CT of the thorax).

\section{Acute vasodilator trial}

As part of their clinical investigations, all patients with $\mathrm{PPH}$ [12] in the institution underwent an acute (therapeutic) vasodilator trial, using adenosine in the cardiac catheter 
laboratory or intensive care unit to assess their suitability for long-term oral vasodilator therapy [13]. Following the placement of radial arterial (Abbocath, Abbott, Ireland) and pulmonary artery (Arrow International, Reading, PA, USA) catheters, a period of 20 min was allowed to reach a haemodynamic steady state. An intravenous infusion of adenosine commenced $\left(5,10,30,50,100,150,200 \mu \mathrm{g} \cdot \mathrm{kg} \cdot \mathrm{min}^{-1}\right.$ [13] via the infusion port of the pulmonary artery catheter, each dose being administered for a period of 3-5 min. Pulmonary and systemic haemodynamic data were recorded at baseline and during steady state conditions at the end of each dosing interval. Pulmonary artery occlusion pressure was estimated following inflation of the catheter balloon, values being taken at end-expiration and averaged over three respiratory cycles. Cardiac output measurements were taken in triplicate, each following an injection of $10 \mathrm{~mL}$ of $5 \%$ dextrose at room temperature, and averaged. Vascular resistances were calculated using standard equations. A positive vasodilator response was defined as a $20 \%$ decrease in pulmonary vascular resistance (PVR). The dose of adenosine was increased until a positive response was obtained, the patient suffered sideeffects (chest discomfort, flushing) or there was a clinically relevant fall in systemic arterial pressure.

\section{Computed tomography protocol}

In all cases, the subjects were placed in the supine position within the CT scanner (Imatron C150L, Imatron Inc., San Francisco, CA, USA). For the purpose of constructing timedensity curves, a rapid multi-section scan acquisition was performed at a single level, immediately prior to, and following the rapid, automated injection $\left(60 \mathrm{~mL}\right.$ at $20 \mathrm{~mL} \cdot \mathrm{s}^{-1}$; Angiomat 6000, Liebal-Flarsheim Company, Cincinnati, $\mathrm{OH}$, USA) of radio-opaque contrast material (Omnipaque $300 \mathrm{mgI} \cdot \mathrm{mL}^{-1}$, Nycomed, Amersham, UK). In each study 15-20, 6-mm sections were obtained. The acquisition time for each section was $100 \mathrm{~ms}$. The interval, between the acquisition of each image was designed to allow construction of complete time-density curves for the lung parenchyma and left-sided circulation (descending aorta). The scans were electrocardiogram-gated and each series was performed during an inspiratory breath-holding manoeuvre. Following completion of the experimental protocol, a calibration study was performed using a bandoleer of extruded acrylic tubes, each containing an incremental concentration $\left(0-18 \mathrm{mg} \cdot \mathrm{mL}^{-1}\right)$ of iodinated contrast. The bandoleer was placed upon foam sections so that it was in the centre of the scanner's reconstruction circle.

\section{Experimental protocol}

Following completion of the acute vasodilator study, patients with $\mathrm{PPH}$ were moved to the CT scanning suite. The patient was left for 15 min to achieve a steady state. At the end of this period, a multi-sequence scan was obtained, $2-3 \mathrm{~cm}$ above the right hemi-diaphragm, during an inspiratory breath-hold manoeuvre. Radiographic contrast was delivered through the side arm of the pulmonary artery catheter. An adenosine infusion was then commenced, at the minimum dose shown to produce a significant fall in PVR in the prior vasodilator study. After a 5-min period, to allow a steady state to develop, a second multi-sequence scan was performed at the original level, during a repeat inspiratory breath-hold manoeuvre. Immediately prior to each multisequence scan, a full set of haemodynamic data, including cardiac output studies, was obtained.
In normal subjects, a single multi-sequence scan was performed in an identical fashion. Radiographic contrast was delivered via an intravenous cannula in a large antecubital fossa vein.

\section{Calculation of perfusion using electron-beam computed tomography}

Using EBCT and following the Sapirstein principle, perfusion can be calculated using equations derived from conventional microsphere approaches to blood flow analysis $[5,14]$ as follows:

$$
\mathrm{PBF} / V=\frac{\Delta \mathrm{Pul}}{\int \mathrm{CDA}, \mathrm{dt}}
$$

Where PBF represents blood flow, $V$ is the unit volume of the lung, $\Delta \mathrm{Pul}$ indicates the peak Hounsfield Unit (HU) change due to contrast material and $\int \mathrm{CDA}$,dt represents the area under the time-density curve for the descending aorta by a gamma-variate fit.

To express blood flow per unit volume of lung parenchyma, it is assumed that the region of interest (ROI) is composed of air and "water" (i.e. blood and parenchyma). The disparate density of these components allows the fraction of each to be calculated using the CT gray scale or Hounsfield number for any ROI. For example, the water fraction can be calculated by subtracting the CT value of pure air from the mean CT value of an ROI to give a value reflecting the amount (density) of parenchyma and blood present in the selected region. Comparing this value to the continuum ranging from water $(0 \mathrm{HU})$ to air $(1000 \mathrm{HU})$ allows the percentage of the ROI that is water (blood and parenchyma) to be calculated as follows [7]:

$$
\text { blood and parenchyma }=\frac{\mathrm{CTROI}-\mathrm{CT}_{\text {air }}}{\mathrm{CT} \text { blood }-\mathrm{CT}_{\text {air }}}
$$

The air fraction of an ROI, is simply one water fraction. Furthermore, the amount of blood present within an ROI can be computed by comparing the time-density curve of the ROI to that of the feeding/draining vessel:

$$
\text { Blood }=\frac{\int \text { CROI,dt }}{\int \text { CDA,dt }}
$$

Where CROI,dt represents the area under the time-density curve of the ROI and CDA,dt the descending aorta.

Subtracting the result from the water fraction produces the percentage of the ROI that is lung parenchyma. Blood flow per unit volume of lung parenchyma can then be calculated by dividing the absolute blood flow per $\mathrm{mL}$ of lung tissue (blood, parenchyma and air) by the fraction of parenchyma in the ROI.

Measurement of cardiac output using EBCT is based on the Stewart-Hamilton equation with iodinated contrast medium used as an indicator [5, 15], as follows:

$$
\text { Flow } \mathrm{L} \cdot \mathrm{min}^{-1}=\frac{\mathrm{K} \times 300 \times \mathrm{V} \times 60}{\mathrm{AUC} \times 1,000}
$$

Where $\mathrm{K}$ represent the constant relating $\mathrm{HU}$ value to iodine concentration $\left(\mathrm{HU} \cdot \mathrm{mg}^{-1}\right), 300$ is the amount of iodine per $\mathrm{mL}$ of contrast $\left(\mathrm{mg} \cdot \mathrm{mL}^{-1}\right), \mathrm{V}$ is the volume of contrast injected (mL), 60 is the correction factor from seconds to minutes, AUC indicates the area under descending aorta time-density curve and 1,000 is the correction from $\mathrm{mL}$ to $\mathrm{L}$.

\section{Image Analysis}

The images obtained were viewed on an off-line workstation and analysed using the scanner's proprietary software. ROI were placed into five regions, at 10, 30, 50, 70 and 
$90 \%$ of the dependent-nondependent lung distance, and time-density curves were constructed for each ROI using a gamma-variate fit to exclude recirculation. Initially the authors had proposed to employ two approaches to calculate perfusion in each sample region, as per previous studies using this technique. First, a single ROI (ROIS) with an approximate sample area of $7-10 \mathrm{~cm}^{2}$ (volume $4-6 \mathrm{~cm}^{3}$; fig. 1a) was employed. In subjects with PPH, when the signal-to-noise ratio was low, multiple estimates of perfusion were obtained $(n=3)$ and the average recorded for that ROI. In a second analysis, a multiple smaller ROI (ROIM) with an approximate sample area of $1 \mathrm{~cm}^{2}$ (volume $0.6 \mathrm{~cm}^{3}$ ) was employed, aiming to avoid all pulmonary vessels (fig. 1b). This second technique could not be applied reproducibly in subjects with $\mathrm{PPH}$, as signal-to-noise ratios were too low. Perfusion was calculated using the technique previously outlined. Relative perfusion, expressed as a fraction of the mean perfusion of the given section, derived using a freehand device to define ROI
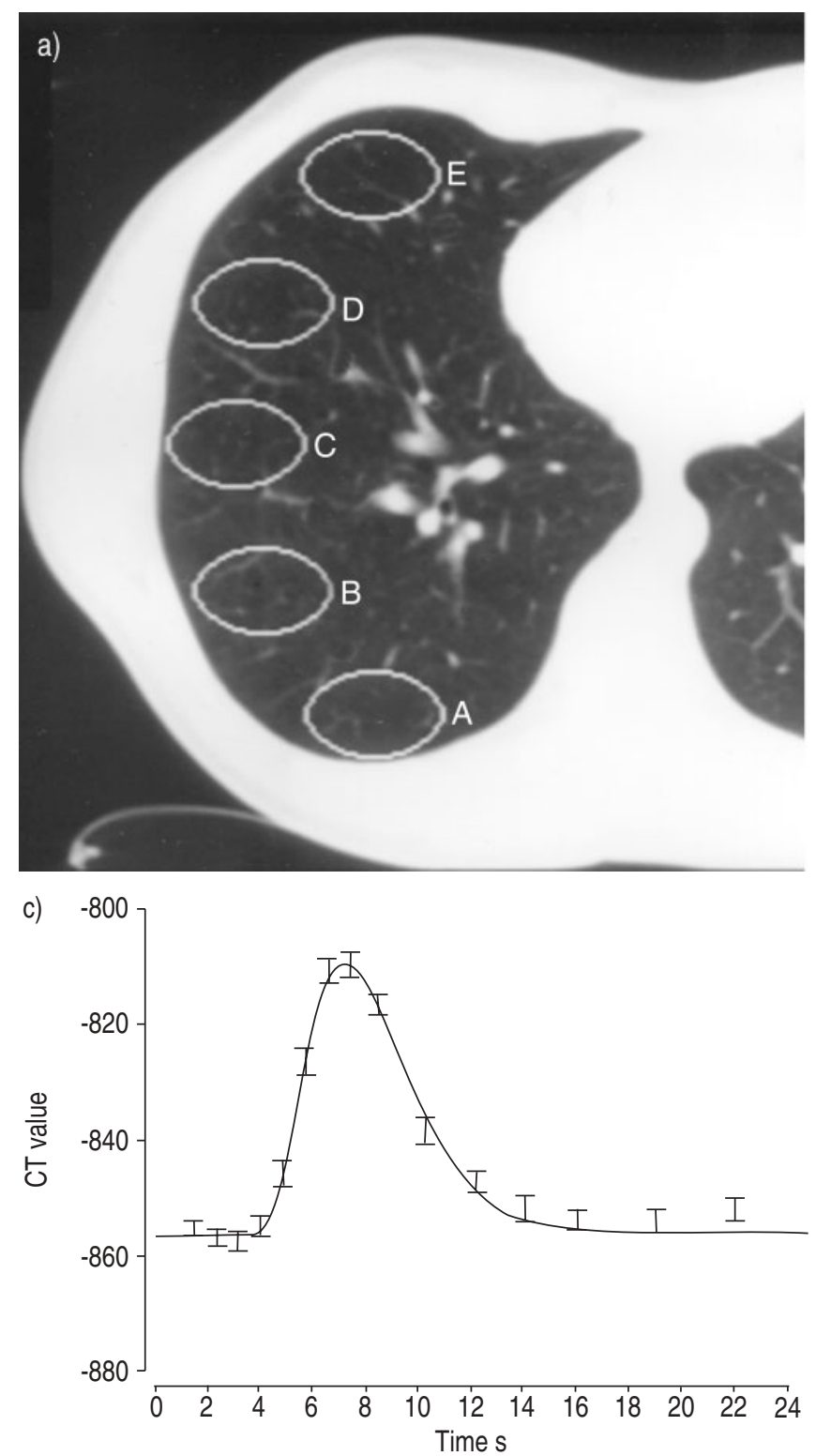

that included the whole of the lung section, except for the hilar and large central vessels, was also calculated.

\section{Statistical analysis}

Comparisons between ventilatory and haemodynamic parameters during each intervention, and differences in regional perfusion (ROIS) under the same study conditions were compared using analyses of variance (ANOVA). For comparisons of perfusion distributions (ROIS) under different study conditions, a two-way ANOVA was applied. A p-value $\leqslant 0.05$ was considered significant.

\section{Results}

Baseline demographic and physiological data for the patients with $\mathrm{PPH}$ are summarised in table 1. All showed a
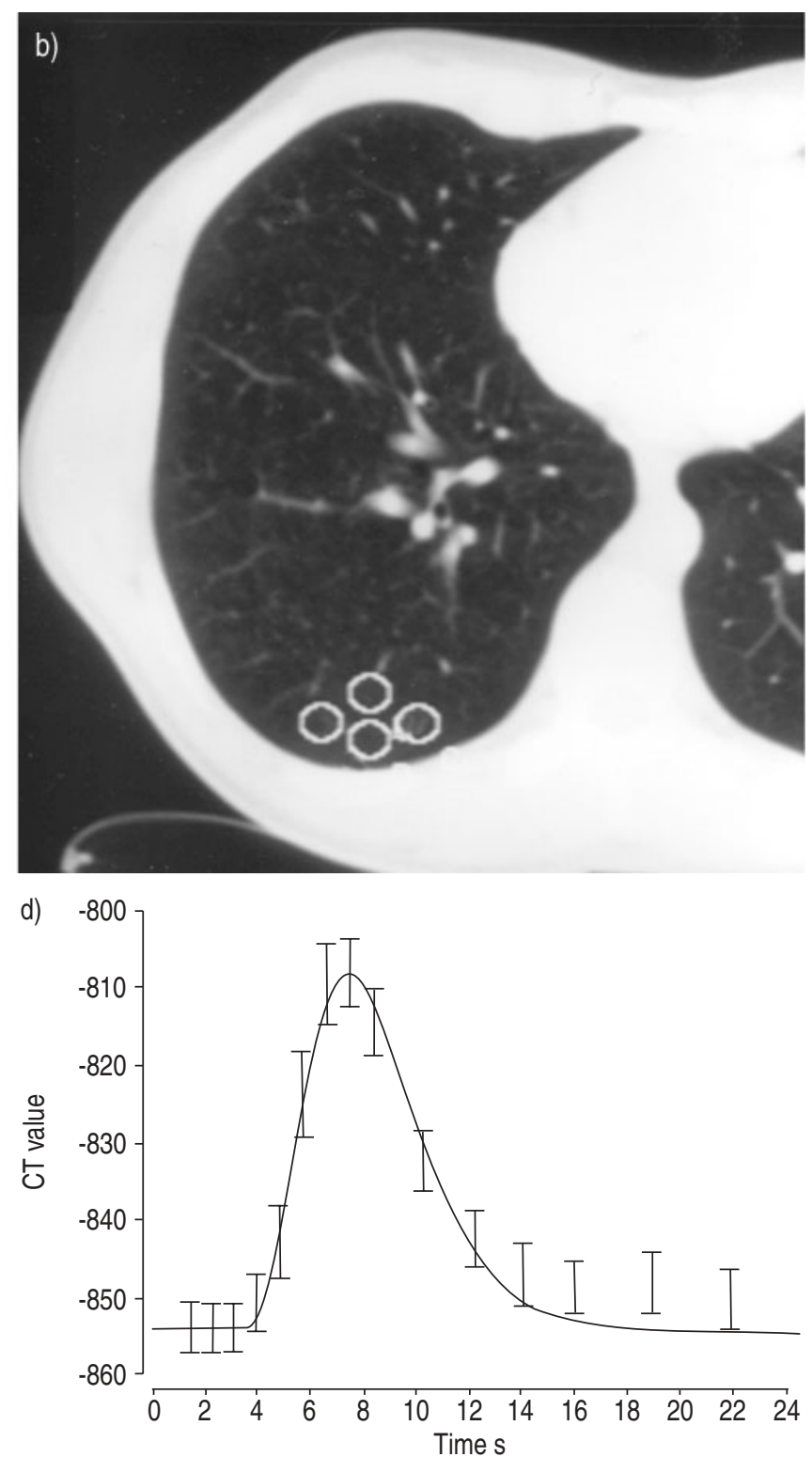

Fig. 1.-Image analysis. a) Single regions of interest (ROI) in the lung section (indicated by white circles) were placed at a) A: 10; B: 30; C: 50; D: 70 E: $90 \%$ of the vertical lung height (normal subjects and subjects with primary pulmonary hypertension). b) Multiple ROI, examining perfusions at $\sim 10 \%$ of the vertical lung height (normal subjects only). c, d) Time/density curves were constructed for each ROI, with a gammavariate fit to exclude re-circulation of contrast. CT: Hounsfield units. 
Table 1.-Baseline demographic and physiological data in subjects with primary pulmonary hypertension

\begin{tabular}{lccccc}
\hline & \multicolumn{5}{c}{ Subject } \\
\cline { 2 - 6 } & 1 & 2 & 3 & 4 & 5 \\
\hline $\mathrm{M} / \mathrm{F}$ & $\mathrm{M}$ & $\mathrm{F}$ & $\mathrm{F}$ & $\mathrm{F}$ & $\mathrm{F}$ \\
Age yrs & 54 & 53 & 61 & 40 & 57 \\
Weight kg & 84 & 49 & 62 & 89 & 65 \\
$\mathrm{CI}$ & 1.57 & 1.95 & 1.59 & 1.3 & 1.49 \\
$P$ pa mmHg & 51 & 59 & 63 & 80 & 64 \\
$\mathrm{PVR} \mathrm{dynes} \cdot \mathrm{s} \cdot \mathrm{cm}^{-5}$ & 2085 & 2640 & 3262 & $\mathrm{NA}^{\#}$ & 1746 \\
$P_{\mathrm{a}, \mathrm{O}_{2}} \mathrm{kPa}$ & 8.4 & 10.1 & 11.6 & 7.83 & 11.1 \\
$\mathrm{~S}_{\mathrm{v}, \mathrm{O}_{2} \mathrm{kPa}}$ & 54 & 65.9 & 65.4 & 54.1 & 59 \\
\hline
\end{tabular}

M: male; F: female; $\mathrm{CI}$ : confidence interval; $P$ pa: mean pulmonary artery pressure; PVR: pulmonary vascular resistance; $P \mathrm{a}, \mathrm{O}_{2}$ : partial pressure of oxygen in arterial blood; $\mathrm{Sv}_{\mathrm{v}} \mathrm{O}_{2}$ : saturation of mixed venous blood. \#: unable to accurately record pulmonary artery occlusion pressure and therefore calculate PVR.

positive vasodilator response to intravenous adenosine, during the trial performed in the intensive care unit. A total of five male, nonsmoking, healthy subjects aged 27-32 yrs were studied. The CT protocol was tolerated well by all subjects and no adverse events were recorded.

\section{Haemodynamics}

The results for the administration of adenosine during the CT protocol, in individual patients with PPH and for the

Table 2. - Physiological data before and during the administration of intravenous adenosine during the computed tomography protocol in individual subjects with primary pulmonary hypertension

\begin{tabular}{|c|c|c|c|c|c|c|c|}
\hline & \multicolumn{5}{|c|}{ Subject } & \multirow[t]{2}{*}{ Mean } & \multirow[t]{2}{*}{ SEM } \\
\hline & 1 & 2 & 3 & 4 & 5 & & \\
\hline \multicolumn{8}{|c|}{$\mathrm{CO} \mathrm{L} \cdot \mathrm{min}^{-1}$} \\
\hline Before & 3.2 & 2.7 & 2.7 & 2.6 & 2.5 & 2.74 & -0.12 \\
\hline During & 4.1 & 3.2 & 3.3 & 2.9 & 3.2 & 3.34 & -0.2 \\
\hline \multicolumn{8}{|c|}{$P$ pa $\mathrm{mmHg}$} \\
\hline Before & 59 & 64 & 67 & 65 & 72 & 65.4 & -2.11 \\
\hline During & 44 & 63 & 55 & 61 & 55 & 55.6 & -3.31 \\
\hline \multicolumn{8}{|c|}{ PVR dynes $\cdot \mathrm{s} \cdot \mathrm{cm}^{-5}$} \\
\hline Before & 1481 & 1275 & 1630 & NA & 1952 & 1584 & -143 \\
\hline During & 1225 & 683 & 1042 & NA & 1100 & 1013 & -116 \\
\hline \multicolumn{8}{|c|}{ MAP $\mathrm{mmHg}$} \\
\hline Before & 116 & 101 & 116 & 79 & 110 & 99.8 & -6.5 \\
\hline During & 96 & 93 & 120 & 85 & 109 & 105.2 & -6.5 \\
\hline \multicolumn{8}{|c|}{ SVR dynes $\cdot \mathrm{s} \cdot \mathrm{cm}^{-5}$} \\
\hline Before & 2050 & 2696 & 3081 & 1867 & 3008 & 2540 & -247 \\
\hline During & 1659 & 2675 & 2521 & 1903 & 2300 & 2212 & -189 \\
\hline \multicolumn{8}{|l|}{$P a, \mathrm{O}_{2} \mathrm{kPa}$} \\
\hline Before & 8.6 & 8.7 & 10.4 & $10.4^{\#}$ & 9.6 & 9.54 & -0.39 \\
\hline During & 8.4 & 10.0 & 9.2 & $10.6^{\#}$ & 9.2 & 9.48 & -0.38 \\
\hline \multicolumn{8}{|c|}{$P \mathrm{a}, \mathrm{CO}_{2} \mathrm{kPa}$} \\
\hline Before & 4.3 & 4.1 & 3.9 & 4.4 & 4.5 & 4.24 & -0.11 \\
\hline During & 4.3 & 3.6 & 3.8 & 4.5 & 4.4 & 4.12 & -0.12 \\
\hline
\end{tabular}

CO: cardiac output; Ppa: mean pulmonary artery pressure; PVR: pulmonary vascular resistance; MAP: mean arterial pressure; NA: not available; SVR: systemic vascular resistance; $P \mathrm{a}, \mathrm{O}_{2}$ : partial pressure of oxygen in arterial blood; $\mathrm{Pa}_{2} \mathrm{CO}_{2}$ : partial pressure of carbon dioxide in arterial blood. \#: received supplemental oxygen (inspiratory oxygen fraction 0.28 ) during scan protocol as increasing hypoxia in dependent posture. group as a whole, are displayed in table 2. Group data revealed an increase in cardiac output $(\mathrm{CO} ; 2.74 \pm 0.12$ versus $\left.3.34 \pm 0.2 \mathrm{~L} \cdot \mathrm{min}^{-1} ; \mathrm{p}=0.06\right)$, with a decrease in pulmonary artery pressure (Ppa; $65.4 \pm 2.11$ versus $55.6 \pm 3.31 \mathrm{mmHg}$; $\mathrm{p}=0.06)$ and a statistically insignificant reduction in PVR $\left(1584.5 \pm 142.5\right.$ versus $1012.5 \pm 116.3$ dynes $\left.\cdot \mathrm{s} \cdot \mathrm{cm}^{-5} ; \mathrm{p}=0.13\right)$. Administration of adenosine resulted in a statistically insignificant increase in mean arterial pressure (MAP; 99.8 \pm 6.5 versus $105.2 \pm 6.5 \mathrm{mmHg}$; $\mathrm{p}=0.13$ ) and a reduction in systemic vascular resistance (SVR; 2540.4 \pm 247 versus 2211.6 \pm 189 dynes $\left.\cdot \mathrm{s} \cdot \mathrm{cm}^{-5} ; \mathrm{p}=0.19\right)$.

\section{Perfusion}

Examination using ROIS revealed a marked nondependentto-dependent gradient in perfusion in normal subjects $(\mathrm{p}<0.01)$, with the maximum perfusion values reaching $75 \%$ of the vertical distance down the lung section. In the lower most regions, perfusion remained level/decreased slightly. By contrast, under baseline conditions, perfusion values in patients with $\mathrm{PPH}$ were significantly lower than those recorded in normal individuals $(\mathrm{p}<0.01)$ and displayed no vertical gradient in perfusion, which was uniform across all ROI ( $p=0.99$; fig. 2). In subjects with PPH, the administration of adenosine resulted in increased perfusion in all ROI $(\mathrm{p}<0.01)$, although the distribution of perfusion remained unchanged ( $\mathrm{p}=0.43$; fig. 3 ). When examining regional perfusion using ROIM in normal individuals, a nondependent-todependent gradient was again detected. Moreover, the use of a high spatial resolution technique confirmed marked perfusion heterogeneity in anatomically close regions of lung (fig. 4).

\section{Discussion}

This study demonstrated a number of important findings. First, when sampled with a large ROI, a gravitational gradient of perfusion was confirmed in the normal lung

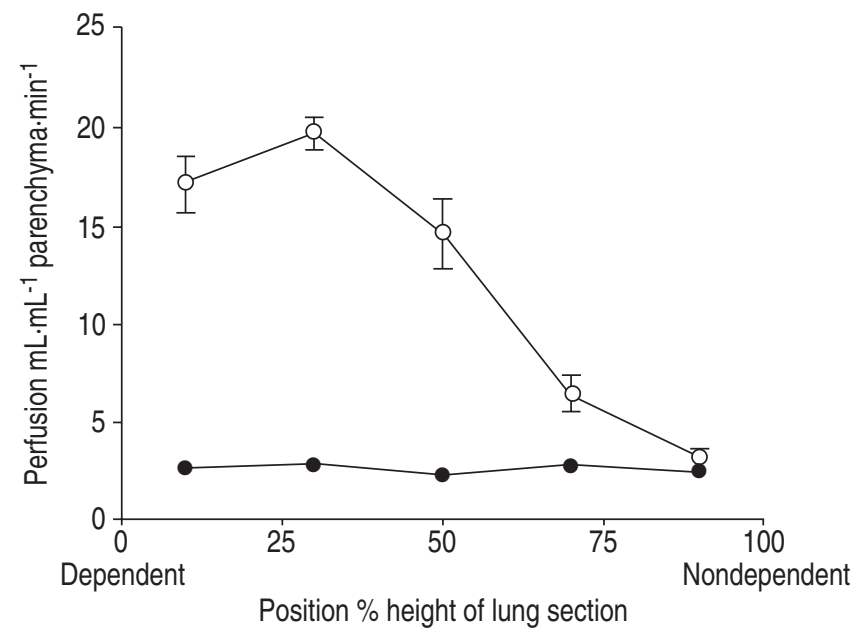

Fig. 2. - Distribution of pulmonary perfusion single region analysis in subjects with primary pulmonary hypertension (PPH; $)$ and normal subjects $(\bigcirc)$. All data are presented as mean \pm SEM. A nondependentto-dependent gradient in perfusion is observed in normal subjects $(\mathrm{p}<0.01)$, with perfusion reaching maximum values of $75 \%$ of the distance down the lung section. In subjects with PPH under baseline conditions, perfusion values were significantly lower than those recorded in normal individuals $(\mathrm{p}<0.01)$ and no vertical gradient in perfusion was demonstrated $(\mathrm{p}=0.99)$. 


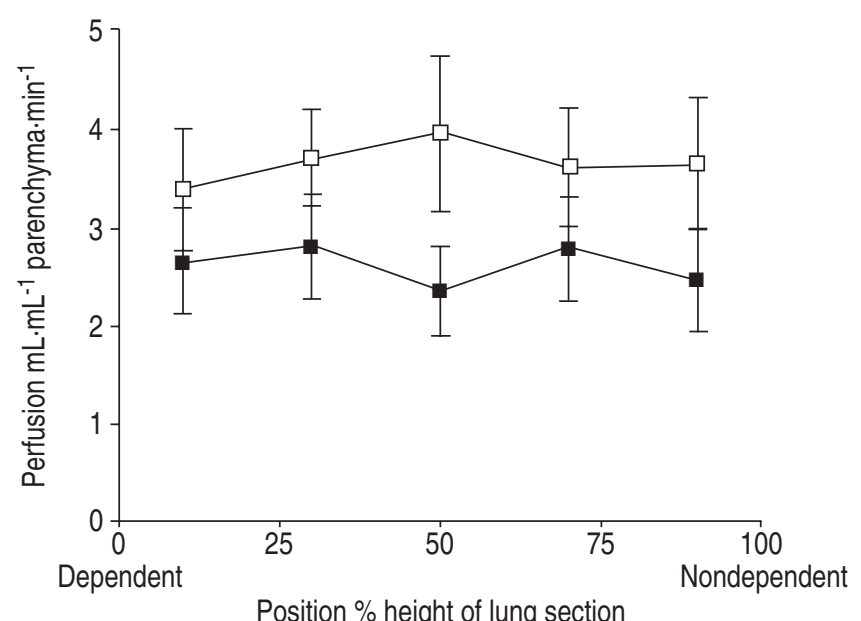

Fig. 3. - The effect of intravenous adenosine on perfusion in subjects with primary pulmonary hypertension. $\mathbf{a}$ : baseline perfusion; $\square$ : during adenosine infusion. The administration of adenosine resulted in increased perfusion in all regions of interest $(p<0.01)$, with the gradient remaining unchanged $(\mathrm{p}=0.43)$.

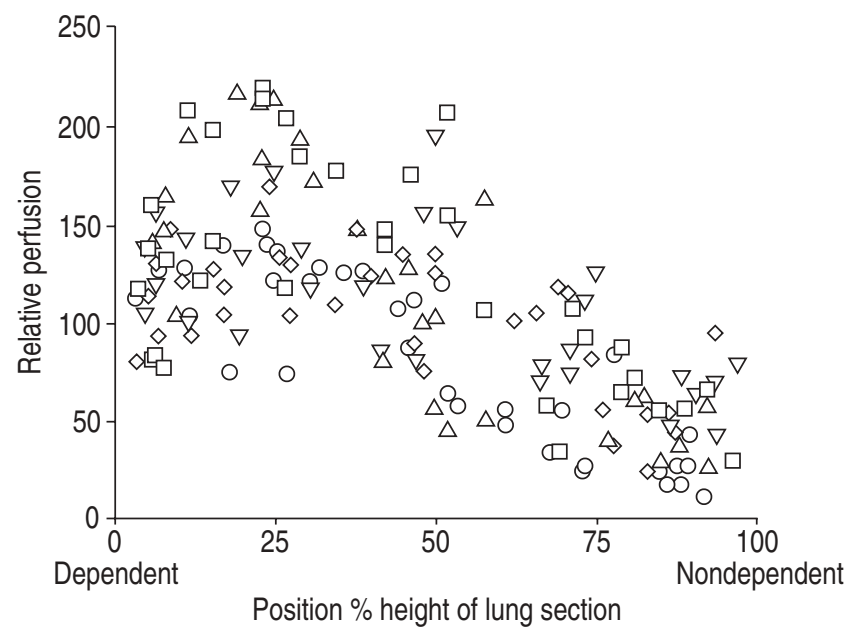

Fig. 4.-Distribution of relative pulmonary perfusion multiple regions of interest in normal subjects. A nondependent-to-dependent gradient was observed. In addition, this relative perfusion data confirms marked perfusion heterogeneity in anatomically close regions of the lung.

using EBCT, which was absent in subjects with PPH. In addition, high spatial resolution analysis confirmed a marked degree of perfusion heterogeneity between anatomically related regions of the lung section in normal subjects. Secondly, pulmonary perfusion values were markedly reduced in subjects in $\mathrm{PPH}$ when compared to normal individuals. Finally, the addition of the nonselective vasodilator adenosine resulted in a generalised increase in pulmonary perfusion. These represent original findings using EBCT and confirm findings obtained using existing techniques.

In the current study, the absolute values of pulmonary perfusion recorded in normal subjects are comparable to previous estimates obtained in humans using this [4] and other techniques [16, 17], and showed (when using a large ROI) a gravitational gradient to perfusion. This is consistent with traditional concepts, which suggest that the distribution of pulmonary perfusion is explained through the interaction of alveolar, pulmonary arterial and venous and interstitial pressures $[1,2]$. In addition, in the same subjects, the use of a higher resolution sampling technique revealed considerable regional perfusion heterogeneity in anatomically close regions of the lungs. This finding is in agreement with the more recent experimental data using high spatial resolution microspheres, which suggest that the branching pattern of the pulmonary vascular tree leads to more heterogeneity of perfusion than could be explained by gravity alone; the so-called fractal hypothesis [3].

By contrast, in subjects with $\mathrm{PPH}$, perfusion levels were markedly reduced and uniform across the lung section. Although there is known to be a reduced alveolar capillary blood volume in PPH [18], no previous studies have recorded absolute perfusion values in either animals or humans. However, the reduced values observed are not unexpected in view of the restricted pulmonary circulation and reduced cardiac output that is a characteristic of this condition.

To date, there have been few studies of regional perfusion in PPH because of the technical difficulties in applying existing techniques to human subjects. Using conventional $\mathrm{CT}$ in subjects with lone pulmonary hypertension associated with systemic sclerosis, the density gradient of the lung between dependent and nondependent regions has been found to be reduced, compared to a similar group with normal pulmonary haemodynamics [8]. The attenuation of the lung, as measured by $\mathrm{CT}$, reflects the physical density of the lungs and is determined by the relative proportions of gas, blood, extravascular fluid and parenchyma. In normal individuals there is an increase in density from nondependent to dependent regions. Possible explanations for this include increased perfusion in dependent regions, the occurrence of micro-atelectasis in dependent regions or a combination of the two. Given that scan acquisition protocols were identical and that parenchymal lung disease had been excluded, the reduction in density gradient seen in those patients with systemic sclerosis and pulmonary hypertension has been interpreted as evidence of a more uniform distribution of perfusion in this group. Pulmonary perfusion scintography was used to investigate the distribution of perfusion in 12 normal subjects and 10 patients with precapillary pulmonary hypertension (eight patients were classified as PPH and two as thromboembolic pulmonary hypertension), in both the supine and upright positions [9]. Perfusion distribution was corrected for lung volume by xenon-133 equilibrium ventilation scans. In both positions, the upper-to-lower zone perfusion ratio was greater in normal subjects compared to patients with precapillary pulmonary hypertension. Using PET to measure regional lung density and blood volume (labelling with ${ }^{11} \mathrm{CO}$ ), the regional distribution of blood volume was found to be abnormally uniform in patients with chronic pulmonary arterial hypertension (five patients with Eisenmenger's syndrome and two patients with PPH) [10]. Traditional models of regional pulmonary perfusion suggest that a more uniform distribution of perfusion is to be expected with pulmonary hypertension $[1,2]$ and in experimental models it has been shown to correlate well with $P$ pa [19]. In this study the mean $P$ pa were high $(65.4 \mathrm{mmHg}$ without adenosine, $55.6 \mathrm{mmHg}$ during adenosine infusion) in the patient group that was studied supine. As such, the vertical lung distance was small. However, normal subjects scanned under the same conditions, displayed a nondependent/dependent gradient in perfusion.

The administration of adenosine during the CT protocol resulted in increases in $\mathrm{CO}$ and reductions in $P$ pa, that just failed to reach statistical significance $(\mathrm{p}=0.06)$, as well as a tendency for a decrease in both SVR and PVR. This is consistent with the effects of a vasodilator given into the central veins, which causes primarily pulmonary vasodilation due to its short duration of action. The haemodynamic response to adenosine in this group of subjects was comparable to that reported by other investigators [11, 20]. Perfusion 
studies revealed a generalised increase in perfusion $(44 \pm 7.7 \%)$ in all ROI, with no change in the regional distribution of perfusion. Adenosine is a vasodilator which acts via intracellular $\mathrm{A}_{2}$ receptors on vascular smooth muscle. When given by central venous infusion, as in this study, it is distributed to all perfused regions of the lungs resulting in the generalised vasodilation observed.

Although the results of this study are in keeping with existing data in both subjects with PPH and normal lungs, a number of limitations in terms of experimental method, are acknowledged. The calculation of perfusion, using EBCT, is based on a combination of indicator-dilution and microsphere algorithms. Previous experimental work in both models and animal studies have revealed that measurement of tissue perfusion using this technique is likely to underestimate true values of perfusion, primarily due to early washout of indicator from the tissue, resulting in reduced peak opacification $[13,21,22]$. This is likely to be exacerbated where the mean transit time of contrast through the tissue is long and therefore give rise to a greater error in subjects with $\mathrm{PPH}$. As such, the difference in perfusion values between normal subjects and those with PPH may have been overestimated. Secondly, radiographical contrast agents, even nonionic media, are known to have mild vasodilatory properties [23, 24]. The relative importance of this effect in the increase in perfusion seen with the addition of adenosine in patients with PPH is difficult to quantify, but in previous work, where subjects have been studied under the same conditions on two occasions, the effects seem to be minimal [4]. Finally, the scanner mode used in this study only allowed perfusion to be calculated in a single slice $(6 \mathrm{~mm})$. Simultaneous, multi-slice imaging, covering up to $8 \mathrm{~cm}$ of the patient, is possible. However, in this format, the resolution of the images and the signal-to-noise ratio is reduced, which would impair the ability to record perfusion, particularly in the lowflow states seen in PPH. Primary pulmonary hypertension represents a diffuse disease process in which all areas of the lung are involved and the authors speculate that the effects seen in one region reflect those in the rest of the lungs. Using this technique, the authors are unable to comment on differences in cephalo-caudal distribution of blood flow between normal individuals and subjects with PPH or in subjects with $\mathrm{PPH}$ following the introduction adenosine.

In conclusion, a vertical gradient of perfusion exists under resting conditions in the supine human lung. In subjects with primary pulmonary hypertension, perfusion values are reduced and perfusion is uniform throughout the lung section. In subjects with primary pulmonary hypertension, the infusion of the vasodilator adenosine led to an increased perfusion in all lung regions. Within the limits discussed, the results of this study are consistent with those cited in the limited literature in this area, as well as with accepted physiological concepts of primary pulmonary hypertension.

\section{References}

1. West JB, Dollery CT, Naimark A. Distribution of blood flow in isolated lung; relation to vascular and alveolar pressures. J Appl Physiol 1964; 19: 713-724.

2. Hughes JMB, Glazier JE, Maloney JE, West JB. Effect of lung volume on the distribution of pulmonary blood flow in man. Resper Physiol 1968; 4: 58-72.

3. Glenny RW, Robertson HT. Fractal modelling of pulmonary flow. J Appl Physiol 1991; 70: 1024-1030.

4. Jones AT, Hansell DM, Evans TW. Pulmonary perfusion in supine and prone positions: an electron-beam computed tomography study. J Appl Physiol 2001; 90: 1342-1348.
5. Wolfkiel CJ, Rich SA. Analysis of regional pulmonary enhancement in dogs using ultrafast computed tomography. Investigative Radiology 1992; 27: 211-216.

6. Larsen RL, Bridges CR, Beck KC, Hoffman EA. Regional pulmonary blood flow via cine $\mathrm{x}$-ray computed tomography. FASEB J 1990; 4: A1074.

7. Tajik JK, Kugelmass SD, Hoffman EA. An automated method for relating regional pulmonary structure and function: integration of dynamic multislice CT and thin slice high-resolution CT. SPIE Proceedings 1993; 1905: 339-350.

8. Cailes JB, du Bois RM, Hansell DM. Density gradient of the lung parenchyma at computed tomographic scanning in patients with pulmonary hypertension and systemic sclerosis. Acad Radiol 1996; 3: 724-730.

9. Horn M, Hooper W, Brach B, Ashburn W, Moser K. Postural changes in pulmonary blood flow in pulmonary hypertension: A noninvasive technique using ventilation perfusion scans. Circulation 1982; 66: 621-626.

10. Wollmer P, Rozkovec A, Rhodes CG, Allan RM, Maseri A. Regional pulmonary blood volume in patients with abnormal blood pressure or flow in the pulmonary circulation. European Heart Journal 1984; 5: 924-931.

11. Nootens M, Schrader B, Kaufmann E, Vestal R, Long W, Rich SA. Comparative acute effects of adenosine and prostacyclin in primary pulmonary hypertension. Chest 1995; 107: 54-57.

12. Rich S. Primary pulmonary hypertension. Executive summery from the World Symposium. Primary Pulmonary Hypertension. World Health Organization, 1998.

13. Rubin LJ. Primary pulmonary hypertension. $N$ Engl J Med 1997; 336: 111-117.

14. Wolfkiel CJ, Fergusson JL, Chomka EV, et al. Measurement of myocardial blood flow by ultrafast computed tomography. Circulation 1987; 76: 1262-1273.

15. Wolfkiel CJ, Fergusson JL, Chomka EV, Law WR, Brundage BH. Determintion of cardiac output by ultrafast computed tomography. Am J Physiol Imaging 1986; 1: 117123.

16. Reed JH, Wood EH. Effect of body position on vertical distribution of pulmonary blood flow. J Appl Physiol 1970; 28: 303-311.

17. Brudin LH, Rhodes SO, Valind SO, Jones T, Hughes JMB. Interrelationships between regional blood flow, blood volume, and ventilation in supine humans. J Appl Physiol 1998; 76: 1205-1210.

18. Borland C, Cox Y, Higgenbottam T. Reduction of pulmonary capillary blood volume in patients with severe unexplained pulmonary hypertension. Thorax 1996; 51: 855-856.

19. Wagner WW. Recruitment of gas exchange vessels. In: Crystal RG, West JB, Weibal ER, Barnes PJ eds. The Lung: Scientific Foundations. Philadelphia Lippencott, Williams and Wilkins, 1997; 1537-1547.

20. Morgan JM, McCormack DG, Griffiths MJD, Morgan CJ, Barnes PJ, Evans TW. Adenosine as a vasodilator in primary pulmonary hypertension. Circulation 1991; 84: 1145-1149.

21. Ludman PF, Darby M, Tomlinson N, Poole-Wilson PA, Rees S. Cardiac flow measurement by ultrafast CT: Validation of continuous and pulsatile flow. J Comput Assist Tomogr 1992; 16: 795-803.

22. Rumberger JA, Feiring AJ, Lipton MJ, Higgins CB, Ell SR, Marcus $M$. Use of ultrafast computed tomography to quantitate regional myocardial perfusion: A preliminary report. J. Am. Coll. Cardiol 1987; 9: 59-69.

23. Peck WW, Slutsky RA, Hackney DB, Mancini GBJ, Higgins CB. Effects of contrast on pulmonary hemodynamics: comparison of ionic and non-ionic agents. Radiol 1983; 149: $371-374$.

24. Higgins CB, Gerber KH, Mattrey RF, Slutsky RA. Evaluation of the hemodynamic effects of intravenous administration of ionic and nonionic contrast materials. Radiol 1982; 142: 681-682. 